\title{
DETERMINATION OF AUTOCORRELATION FUNCTION OF WOVEN FABRICS BY USING LASER SPECKLE
}

\author{
Eiji ToBA \\ The Faculty of Textile Science and Technology, Shinshu University
}

\section{INTRODUCTION}

It is known that when a rough surface is illuminated by a coherent light such as a laser, a reflected or a transmitted light forms a diffraction fringe on the observation plane. This fringe is called speckle pattern. The speckle pattern is a diffraction pattern caused by the random phase of light reflected or transmitted at the surface. Therefore it contains the information about the surface state of the irradiated material.

The studies ${ }^{1-5}$ ) on the optical mechanism of the formation of the speckle pattern and its statistical characteristics have been reported, but very few of them have applied the variation in the speckle pattern to the practical measurements. ${ }^{6) ~ 9)}$ Only a few studies applied it to analyze the vibration or to detect dynamically the displacement of diffused plane.

In the present study, as the rough surface, woven fabrics having twodimensional or binary lattice configurations were investigated. Autocorrelation functions of the woven fabrics were examined ${ }^{10)}$, and also, the experiments ${ }^{11}$ ) 12) for discrimination of the texture and to detection of the defects in the fabrics were performed by utilizing the speckle pattern obtained from the reflected or the transmitted light by laser beam irradiation.

\section{THEORY}

For a rough body (object plane) being irradiated by a coherent light, the relation between the power spectrum of the speckle pattern which is obtained by the reflected or the transmitted light and whose intensity distributions at the object plane is described follow.

One-dimensional analyses were used for convenience.

As shown in Fig. 1, $u, x$, and $R$ represent the coordinates of the object plane, the observation plane for speckle pattern (a photographic film is used as the observation plane) and the distance of the respective planes. The laser 


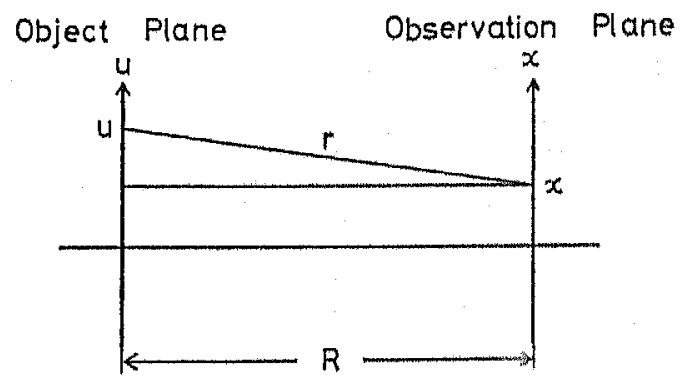

Fig. 1 Coordinate pertinent to speckle pattern analysis

beam with wave length $\lambda$ is irradiated for an object. When the intensity distri bution of the light reffected or transmitted at object plane define as $f(u)$, the amplitude distribution of its is given by the form $I^{\frac{1}{2}}(u) e^{i \omega(m)}$. Where $\psi(w)$ ta kes values of between $-\pi$ to $\pi$ at random and uniformly, and $\phi(u)$ is a real function which varies rapidly compared with $I(u)$. The amplitude distribution $A(x)$ of the reflected or transmitted light at point $x$ on the observation plane is given by

$$
A(x)=\frac{1}{\lambda R} \int \Gamma^{\frac{1}{2}}(u) e^{i \phi(u)} e^{\frac{i 2 n+x}{\lambda}} d u
$$

Therefore, assuming the $R$ is large enough in comparison with $|u-x|$ in the term $r=\sqrt{(u-x)^{2}+R^{2}}$, this assumption can be considered as the condition for Fraunhofer's diffraction, then $r \cong R+(u-x)^{2} / 2 R$, the intensity distribution $|A(x)|^{2}$ can be written in the form

$$
|A(x)|^{2}=\frac{1}{\lambda^{2} R^{2}} \iint\{I(u) I(u+\tau)\}^{\frac{1}{2}} e^{i \psi(u, \tau)} e^{-i D r\left(u-x+\frac{\tau}{2}\right)} d u d \tau
$$

where putting $p=2 \pi / \lambda R$, since the incident beam is monochromatic, the phase difference between each the light diffracted at the points $u$ and $\nu$ on object plane is fixed. Therefore, as following relation holds,

$$
I^{\frac{1}{2}}(u) e^{2 \pi i \psi(u)} I^{\frac{1}{2}}(v) e^{-2 \pi i \psi(v)}=\{I(u) I(v)\}^{\frac{1}{2}} e^{2 \pi i \psi(u, v)}
$$

$\tau=u-v, \phi(u)-\psi(v)=\psi(u, \tau)$ are established.

The intensity distribution $|A(x)|^{2}$ is recorded on the photographic film at the observation plane. This film is irradiated successively by laser beam with wave length $\lambda^{\prime}$ and is subjected in Fourier transformation, through the optical system as shown in Fig. 2. Suppose the amplitude transparence for the photographic 


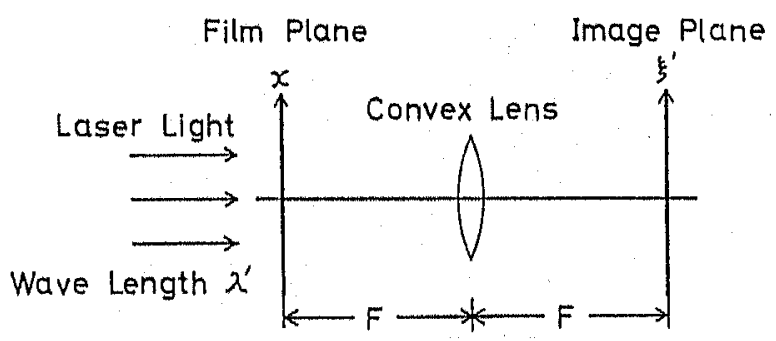

Fig. 2 Fourier transform optical system

film is proportional to the intensity distribution of light, $F$ being taken to be a focal distance, intensity distribution $P\left(\xi^{\prime}\right)$ at the image plane in Fig. 2 is given by

$$
\begin{aligned}
P\left(\xi^{\prime}\right) & =\left.\left.\left|\int\right| A(x)\right|^{2} e^{-i q \xi^{\prime} x} d x\right|^{2} \\
& =C \iint\left(I(u) I(v) I\left(u+\frac{q}{p} \xi^{\prime}\right) I\left(v+\frac{q}{p^{\prime}} \xi^{\prime}\right)\right\}^{\frac{1}{2}} e^{i\left(\psi\left(u, \frac{q}{b} \xi^{\prime}\right)-\psi\left(v, \frac{q}{b} \xi^{\prime}\right)\right\}} d u d v
\end{aligned}
$$

where $2 \pi / \lambda^{\prime} F=q$, and $u=v$ in the term $e^{i\left\{\psi\left(u, \frac{q}{b} \xi^{\prime}\right)-\psi\left(v, \frac{q}{p} \xi^{\prime}\right)\right\}}$

becomes unity, however it converges to zero in other conditions of $u \neq v$. Consequently, $P\left(\xi^{\prime}\right)$ is expressed as eq. (5)

$$
P\left(\xi^{\prime}\right)=C \int I(u) I\left(u+\frac{q}{p} \xi^{\prime}\right) d u
$$

Putting $(q / p) \xi^{\prime}=\xi$ in eq. 5 , eq. 6 is obtained

$$
P\left(\frac{\lambda^{\prime} F}{\lambda R^{\xi}}\right)=C \int I(u) I(u+\xi) d u
$$

It is noticeable that the power spectrum of speckle pattern is identical with the autocorrelation function magnified by a factor of $\lambda^{\prime} F / \lambda R$ as refered to the original scale in object plane. As the test material, if the woven fabrics with binary lattice configuration use and by determining autocorrelation function of them it is possible to obtain certain information of their surface static states and planer yarn configurations. Thus the speckle pattern can be applied to discriminate the textile structures. Generally the autocorrelation function of the material which maintains periodic regularity in configuration is appeared distinctly or sharply, however for irregular, the autocorrelation function becomes to be indistinct and its peaks lose sharpness. Namely, the variation or the degree of shading in pattern of autocorrelation function makes it possible to 
a)

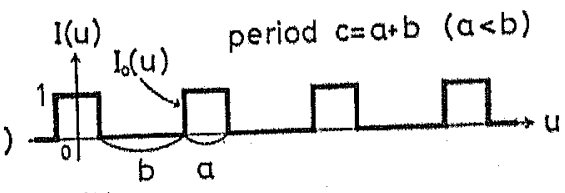

b)

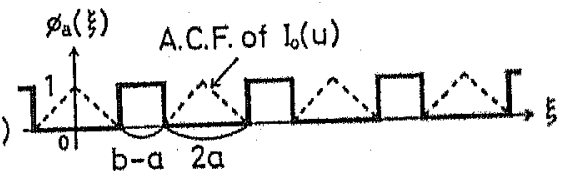

c)

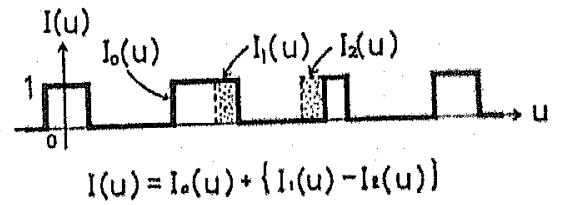

Fig. 3 Binary periodic function and its autocorrelation function

assess the existence of defects in materials. The binary periodic function $l(\boldsymbol{u})$ $=I_{0}(u)$ as shown in Fig. 3(a) is considered as follows, $a$ is the pulse width, $b$ is the pulse interval and $c$ is the period respectively. If the function $I(u)$ is transformed to eq. (7) with the variation of period

$$
I(u)=I_{0}(u) \pm\left\{I_{1}(u) \pm I_{2}(u)\right\}
$$

The original function $I_{0}(u)$ is modified by both $I_{1}(u)$ and $I_{n}(u)$. An example is shown in Fig. $3(\mathrm{c})$. The autocorrelation function $\phi_{a}(\xi)$ obtained from $I(u)$ is given by

$$
\phi_{a}(\xi)=\phi_{00}(\xi)+\phi_{11}(\xi)+\phi_{22}(\xi) \pm \phi_{01}(\xi) \pm \phi_{02}(\xi) \pm \phi_{12}(\xi) \pm \phi_{10}(\xi) \pm \phi_{20}(\xi) \pm \phi_{21}(\xi)
$$

where $\phi_{00}(\xi)$ is the autocorrelation function of the original function $\nu_{0}(u)$. The modification from original relationship of $I(u)=I_{0}(u)$ to eq. $(7)$ adds crosscorrela . tion function components in autocorrelation function as given in eq. (8), and changes its pattern.

By setting the filter which cut off the light exhibiting a similar distribution as $\phi_{00}(\xi)$ shown by dotted line in Fig. $3(\mathrm{~b})$ and by measuring the intensity of transmitting light at the image plane of autocorrelation function, the variation in $I(u)$ can be detected. Thus the defects of woven fabrics can be detected by the evaluation of the degree of shading in autocorrelation function obtained from woven fabrics.

\section{EXPERIMENTAL}

\subsection{Test materials}


The trial products of plane weave, twill weave and satin weave whose preparation procedures were previously known were used to determine the textures of woven fabrics. The warp fineness, filling fineness, thread density and structure of the test materials are shown in Table 1 . In the structural diagrams for test fabrics shown in Table 1, warp and filling yarns respectively turn their vertical positions at the boundary lines between bright squares and dark ones,

Table 1

\begin{tabular}{|c|c|c|c|c|c|c|}
\hline $\begin{array}{c}\text { Textile } \\
\text { Weave }\end{array}$ & Warp & Filling & Warp & Filling & $\begin{array}{c}\text { Thickness } \\
\text { (mm) }\end{array}$ & $\begin{array}{c}\text { Textile } \\
\text { Design }\end{array}$ \\
\hline $\begin{array}{c}\text { Plane } \\
\text { Weave }\end{array}$ & 82 & 110 & 52 & 32 & 0.10 & Threads Per cmes \\
\hline $\begin{array}{c}\text { Twill } \\
\text { Weave }\end{array}$ & 72 & 138 & 48 & 39 & 0.15 & \\
\hline & & 83 & 80 & 40 & 0.12 & \\
\hline $\begin{array}{c}\text { Satin } \\
\text { Weave }\end{array}$ & 42 & & & & \\
\hline
\end{tabular}

Two model samples were designed to detect the defects in woven fabrics. One is a photographic film sample printed the grating pattern providing two alternative transmission distribution coefficients. The other is a grating plate prepared by mounting cotton yarns (fineness; 796 de) in parallel with $1 \mathrm{~mm}$ division.

As the defective woven fabric samples, PET plane weaves having a yarn break, and a hole of about $0.5 \mathrm{~mm}$ square were mainly used. The color of woven fabrics and cotton yarns used was white only.

\subsection{Experimental method}

The experimental apparatus configurations for obtaining the speckle patterns from a reflected and a transmitted light respectively show in Fig. 4(a), Fig. 4(b). Apparatus (1) shows the He-Ne gas laser $(5 \mathrm{~mW}$ output power, wave length $\lambda=6328 \AA$ ). Laser beam is expanded and collimated through objective lens (2), pinhole (3) and collimating lens (4). The collimated laser beam with the diameter of the aperture kept in $15 \mathrm{~mm}$ (5) and is irradiated the woven fabric surfaces (7) $(21 \mathrm{~mm} \times 16 \mathrm{~mm}$ in size). The speckle patterns are obtained by the reflected 


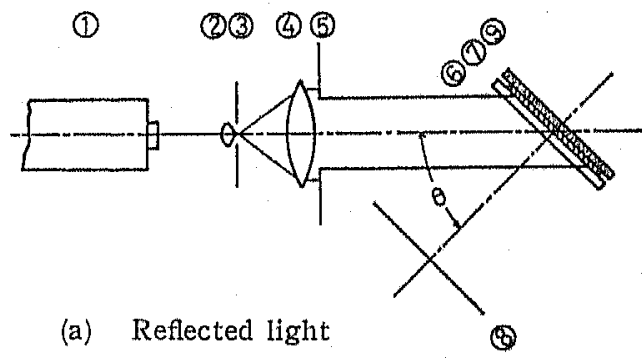

(1) He-Ne Gas Laser

(2) Objectiv Lans

(3) Pinhole

(4) Collimating Lens

(2) Aperture

(6) Transparent Glass

(t) Object

(8) Photographic Film

(9) Acryl Plate (black)

0 Ground Glas

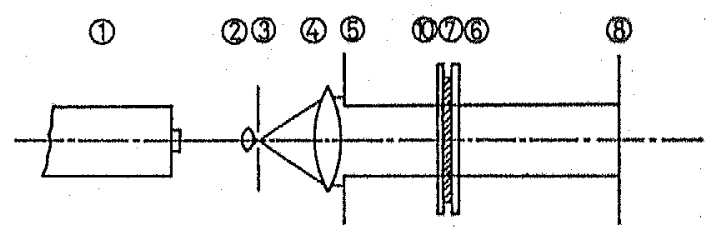

(b) Transmitted light

Fig. 4 Experimental arrangement for obtaining speckla pattern

light from the fabric surface and by the transmitted light through the fabrics, the speckle patterns are recorded on the photographic film (Fuji Film Co. MINICOPY). The fabrics are fitted with clean glass plate (6) (1mm thickness) and blackened acryl plate (9) (1 $\mathrm{mm}$ thickness). Frosted glass (10 (1mm thickness) allow the light from the fabric surface to be taken place a sufficient phase variation. The fabrics are mounted as their warp yarns being set upon the equatorial plane of the systems in Fig. 4(a) and Fig. 4(b). The distance between the film surface and that of the fabric is $150 \mathrm{~mm}$. In reflection method, the incident angle $\theta$ of the laser beam for the fabric surface is 45 degree.

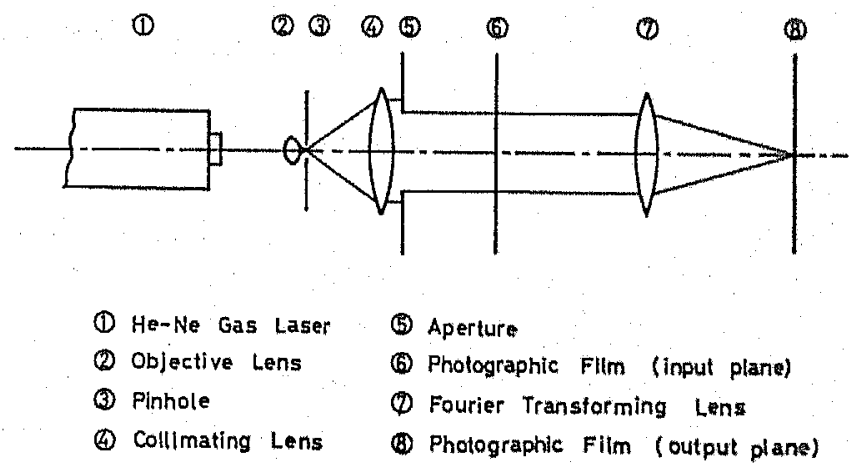

Fig. 5 Experimental arrangement for obtaining power spectrum of speckle pattern 
Exposing time is $0.25 \sim 1$ sec.

The assembly for recording the power spectrum (autocorrelation function) obtained from the speckle pattern of fabrics is shown in Fig. 5. He-Ne gas laser beam ( $5 \mathrm{~mW}$ output power, wave length $\lambda^{\prime}=6328 \AA$ ) is expanded and collimated into $15 \mathrm{~mm}$ in diameter and irradiated the film (6) which previously photographed the speckle pattern. The speckle pattern is subjected in Fouriertransformation through lens ( $)$ (forcal length $F=300 \mathrm{~mm}$ ). Then the autocorrelation functions of the fabrics are recorded on the photographic film place at the focal plane (8) of lens (7). The assembly for detecting the defects in fabrics is shown in Fig. 6. The filter is placed correctly on the image plane (8) of the

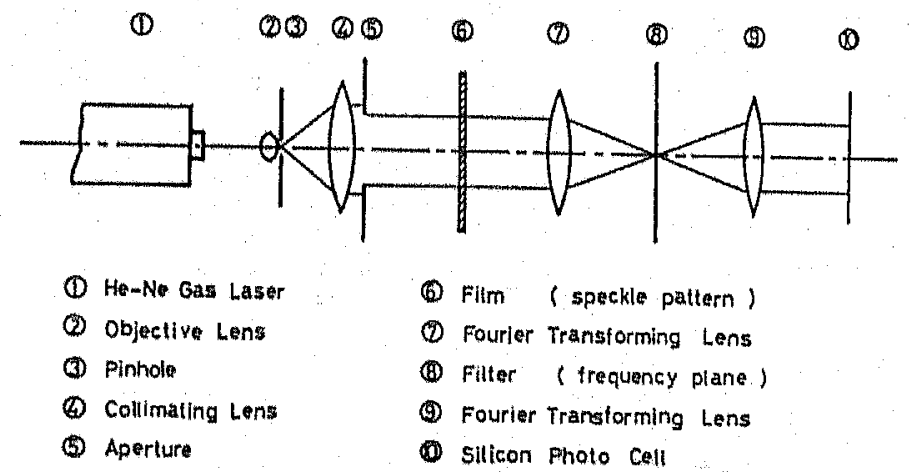

Fig. 6 Experimental arrangement for detecting defect of fabrics

autocorrelation function of fabrics in order to allow the amplitude transmission level maintain a value of zero or unity alternatively. Quantity of the transmitted light is received by silicon photo-electric cell, and also, photoelectromotive force $V$ is measured. For correcting the defect of photographical contrast variation on photo-electromotive force $V_{0}$, which is measured without the filter as well. The values of $r$ given in term of $r=V / V_{0}$ are determined.

\section{EXPERIMENTAL RESULTS AND DISCUSSION}

\subsection{Autocorrelation function of woven fabrics}

Autocorrelation function patterns by the reflected and the transmitted light obtained from different three fundamental textile weaves such as plane weave, twill weave and satin weave are shown in Fig. 7 respectively. The periods for the direction in warp $(\$)$, in filling $(\leftrightarrow)$ and in bias $(\not x)$ which determined from the original weave density are compared with those are evaluated from their autocorrelation function in the original scale of weaves and are shown in 


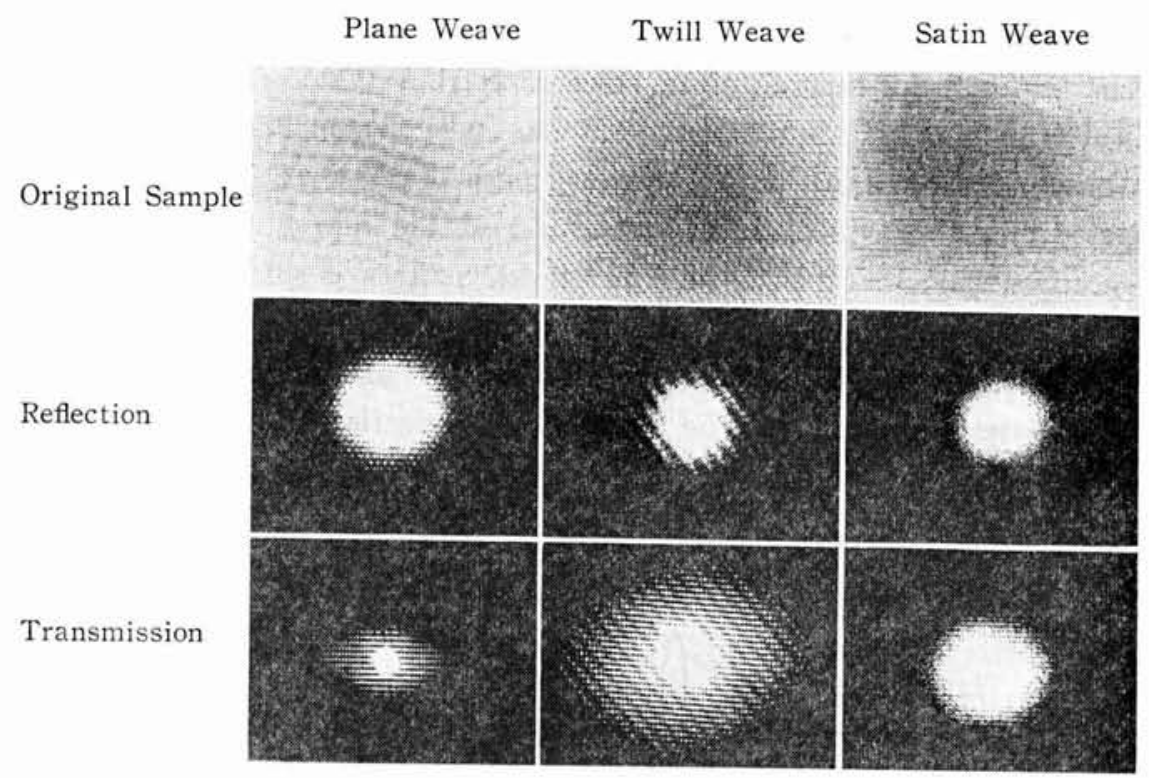

Fig. 7 Autocorrelation function of fabrics

Table 2

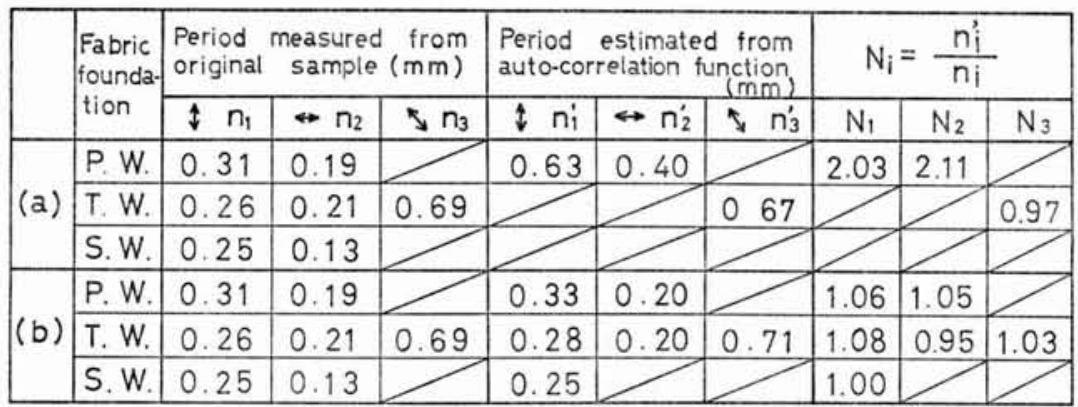

(a) Reflection (b) Transmission

Table 2. As shown in Table 2, the periods obtained from the original weave density completely agreed with the autocorrelation functions. The autocorrelation function of plane weaves was found to consist of only two periodic components in $(\imath)$ and $(\leftrightarrow)$ directions. Especially according to the reflection, the periods evaluated from an autocorrelation function in individual $(\hat{\imath})$ and $\leftrightarrow$ ) directions were twice those calculated from an original woven fabric. This result is considered in terms of the periodic components of the float intervals associated with the configuration of both the warp and filling yarns in the plane weave. 
Twill weave consist of the periodic components not only in $(\uparrow)$ and $(\leftrightarrow)$ directions, but in direction $\left(x^{7}\right)$. In reflection method, marked periodic com ponent which observed in direction $(\not)$ is characterized by the structure of the twill weave. In observation of the autocorrelation function for the twill weave, by assuming $a, b$, and $c$ to be the peak intervals traveling in the directions of $(\downarrow),(x)$ and $\left(x^{x}\right)$ respectively, and by putting $\psi$ to be the gradient angle between the bright fringe lines and equatorial line. Following empirical formula is obtained.

$$
K=\frac{c}{2}\left(\frac{1}{a \cos \psi}+\frac{1}{b \sin \psi}\right)
$$

Consequently, by determining the value for $K$ using by eq. 9 , it is possible to evaluate the original structure of the twill weave.

Satin weave is characterized by less distinguishability among its periodic components in the directions of $(\uparrow),(\leftrightarrow)$ and $\left(\chi^{\star}\right)$. It is considered to coincide with the facts that the satin weaves have a small number of intersections by the two threads of warp and filling ones as shown in Table 1 , and have little threads interstice since its threads are used of fine.

As mentioned above, the autocorrelation function of the woven fabrics informed their morphological characteristics because it is varied depending on the structures of them. In other words, the determination of this function allowed us to get the informations about surface morphologies of woven fabrics in reflection method, and also, in transmission method it allowed us to gain them about the planer configurations of fabrics.

Thus it is possible to discriminate between three fundamental weaves.

\subsection{Autocorrelation function of model samples and defective weares}

A

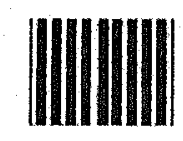

$B$

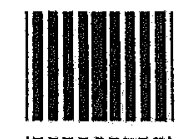

C

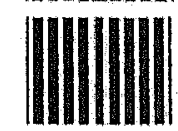

D

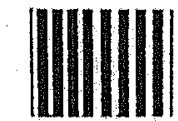

Fig. 8 Model samples and its transmission function

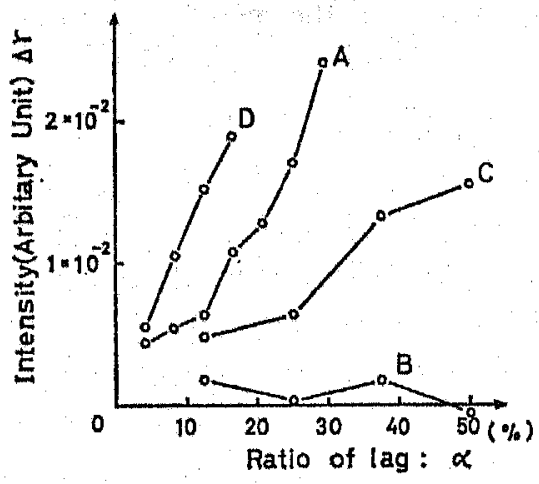

Fig. 9 
Based on the consideration of various practical defects in woven fabrics. model samples bearing binary transmission distribution were produced by the help of photographic films. Designs of the model samples and their transmis. sion functions are shown in Fig. 8. Four different grating patterns were printed on the sample films individually. In sample $A$, every grating stripe was $2 \mathrm{~mm}$ in width, but the central one had its position shifted toward the left. Namely the grating periods (intervals) at both of its sides were therefore varied.

In samples $B$ and $C$, grating periods were fixed in $1 \mathrm{~mm}$, however its central stripe was made wider in $B$, narrower in $C$, than of others. Yarn break in defective fabric was understood to be the particular case in the modes sample C. In sample D, the grating stripe width was fixed in $2 \mathrm{~mm}$ and the grating periods were continuously varied. This sample $D$ corresponded to the defective fabric with dispersive weave density, that, is with weaving bar.

Relations between the sizes of defects and $r$ values are shown in Fig. 9. Where the horizontal axis represented the index $\alpha$ of magnitude of the defects in percentage, expressed by either the ratio of periodic las for the samples $A$ and $D$ or the ratio of width variation for the samples $B$ and $C$.

Samples $A, C$ and $D$ provided the linear proportional relationships between the values of $\alpha$ and $r$. Sample B made the terms above the second in eq. (7) and those above the fourth in eq. (8) be negative, and made the autocorrela. tion functions $\phi_{14}(\xi), \phi_{22}(\xi)$ lay within the region of $\phi_{00}(\xi)$. Accordingly, the quantitative variation in the light passing through the filter not vary quant: tatively. In other words, since the autocorrelation function of the sample $B$ was brought within the spacial cut off frequency range of the filter, the defects in the sample $B$ could not be detected. For the samples with periodic shift $(A$. D), variation in $r$ value was greater than those with variation width (B, C). It was suggested that the method established in this study to measure the characteristics of the practical woven fabrics was superior to double diffraction method, because it has made it possible to detect the defects in fabrics with a lesser influence of thread morphology (i. e. yarn evenness, fuzzing).

Autocorrelation functions and $d r^{*}$ values obtained from the cotton yarn grating model with $1 \mathrm{~mm}$ regular periods (intervals) and from those with various, irregurated periods are shown in Fig. 10. The model with $1 \mathrm{~mm}$ periods, with about $10 \%$ periodic lag and with about $50 \%$ periodic lag respectively are shown in Fig. 10 (a), (b) and (c).

The autocorrelation functions and $\Delta r$ values of original PET plane weave and defective PET weaves are shown in Fig. 11. In Fig. 11, (a), (b), and (c) shows respectively the original normal weave, defective one with a hole of 
a)

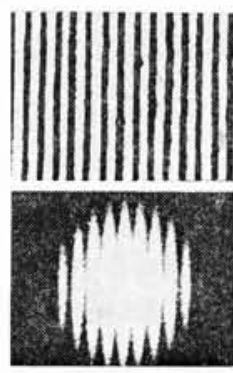

Original

Fig. 10 (b)

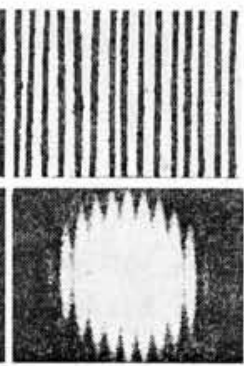

$\Delta \gamma=6.4 \times 10^{-3}$

(b)

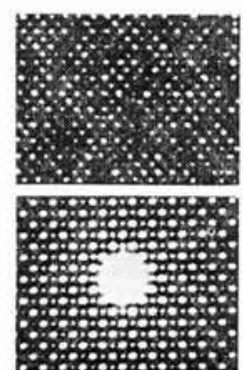

Original

Fig. 11
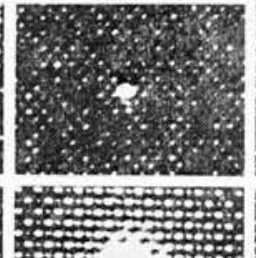

$\Delta \gamma=5.6 \times 10^{-3}$

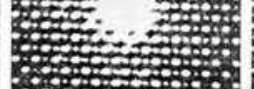

(c)

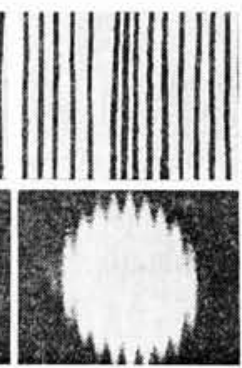

$\Delta \gamma=10.6 \times 10^{-3}$

PET Plane Weave

A. C. F.
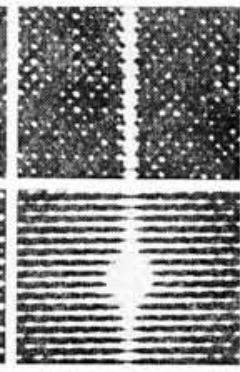

$\Delta \gamma=17.7 \times 10^{-3}$

(c) ,

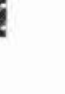


long time in determination process.

\section{CONCLUSION}

The results obtained in this study can be summarized as follows. Autocor* relation functions of the static woven fabrics are obtained by utilizing laser speckle pattern. The autocorrelation functions indicate the following facts.

1) By the reflection method, the surface configuration of a woven fabric can be known, on the other hand, by the transmission, the state of plante arrangement of yarn can be known.

2) Three fundamental weaves, i. e. plane weave, twill weave, and satin weave, can be discriminated.

3) The presence of periodic defect in the woven fabric and its size can bo detected.

\section{ACKNOWLEDGMENT}

The author is pleased to acknowledge the considerable assistance of Professor M. Sawaji.

\section{REFERENCES}

1) L. I. Goldfischer, Jour, Opt. Soc, Amer, Vol. 50, No. 3, 247/253 [1965)

2) T. Asaturu et al., Optica Acta, Vol, 19, 274 (1972)

3) T. Suzuri et al., Japan. J. Appl. Phys, Vol, 5, No, 9, 807/813 (1966)

4) R. B. Crann, Jour. Opt. Soc. Amer., Vol. 60, No, 12, 1658/1663 (1970)

5) J. C. DANTy, Optica Acta, Vol, 17, No, 10, 761/772 (1970)

6) J. Tsujiuchi et al., Optic, Vol. 3, No. 3, 166/176 (1974)

7) H. J. TrziAnI, Optica Acta, Vol, 18, No. 12, 891/902 (1971)

8) N. Ferneluus et al., Jour. Opt. Soc. Amer., Vol. 61, 556/572 (1971)

9) J. A, Leendertz, J. Sci, Instrum., Vol. 3, 214 (1970)

10) E. Tова et al., Semi-Ann. Meet, Soc. Instrum, \& Control Engra. Japan, Preprint, 547/548 (1975)

11) E. ТовA et al., Japan, Sen-i Gakkaishi, Vol, 33, No. 9, 83/89 (1977)

12) E. ToвA et al., Semi-Ann. Meet., Soc. Fiber Sci, \& Techn, Japan, Preprint, $78 / 81(1976)$ 